\title{
A New MIMO ANFIS-PSO Based NARMA-L2 Controller for Nonlinear Dynamic Systems
}

\author{
Yousif Al-Dunainawi ${ }^{1}$; Maysam F. Abbod ${ }^{2}$ and Ali Jizany ${ }^{3}$ \\ ${ }^{1,2}$ Electronic and Computer Engineering Dept. \\ College of Engineering, Design and Physical Sciences \\ Brunel University London \\ Uxbridge, London, UK \\ yousif.al-dunainawi@brunel.ac.uk, \\ ${ }^{3}$ Applied Computing Department \\ Buckingham University \\ Buckingham, UK \\ ali.jizany@buckingham.ac.uk \\ maysam.abbod@brunel.ac.uk
}

\begin{abstract}
The proposal of this study is a new nonlinear autoregressive moving average, NARMA-L2 controller, which is based on an adaptive neuro-fuzzy inference system, ANFIS architecture. The new control configuration employs Sugeno-type fuzzy inference system FIS submodels to map input characteristics to the output of a dynamic and nonlinear system. The default hybrid learning algorithm (Backpropagation and Least Square Error) has been carried out as well as particle swarm optimisation (PSO) approach, in order to select the optimal parameters of the ANFIS submodels. Once the system has been modelled efficiently and accurately, the proposed controller is designed by rearranging the generalised FIS submodels. The controller performance is evaluated by simulations conducted on a binary distillation column, which is characterised by a nonlinear and dynamic behaviour. The obtained results show that the PSO-ANFIS based NARMA-L2 achieved more efficient modelling and control performances when compared with other controllers. These controllers include ANN-based NARMA-L2, (PD, PI and PID like) fuzzy-tuned by GA and PSO and traditional PID, which are also implemented to the column for comparison. Stability and robustness of the proposed controller regarding system inputs variance have also been tested by applying asynchronous setpoints of both inputs of the process.
\end{abstract}

Keywords: Intelligent control; ANFIS; NARMA-L2; Nonlinear systems; Fuzzy control.

\section{Introduction}

As a result of the worldwide ambition for more reliable attainment of high product quality, more efficient use of energy, tighter safety and environmental regulations, industrial processes have evolved over recent years into very complex, highly nonlinear and integrated systems [1].

Rigorous demands like these naturally lead to more difficult and challenging control problems for today's industrial control engineers; problems requiring more efficient solutions than can be achieved by only conventional techniques. It also required inter- and cross- 
disciplinary research, development, as well as collaboration in both industry and academia. Cooperation between control and other disciplines has been consistently fruitful [2].

A big drive has been seen in the academic community to design new control systems, either by traditional or contemporary methods. Introducing an intelligent control system can be the key factor in improving performance as well as deal better with challengeable features of nonlinear and complex processes, although linear-based control systems are frequently used. In general, reasonable performance is attained over a narrow operating range, however, when a wide range of process tasks is a prerequisite, the nonlinearities become more critical, and the control performance is sacrificed [3].

Intelligence based methods emerged two decades ago to act as an effective solution in many applications, many of comprehensive reviews had been written showing that its importance and widespread applications [4]-[6]. From a control viewpoint, when nonlinearity, uncertainty or control difficulties result from dynamic processes, which may bring severe complications to analysis and synthesis. The most applied approaches such as Artificial Neural Networks (ANNs), Fuzzy Logic (FLC), is an essential 'intelligent techniques' approach which is employed considerably in order to control nonlinear processes. One of the strongest arguments for the use of fuzzy based controllers is their ability to exploit the tolerances for uncertainty and nonlinearity, in order to achieve robustness and controllability, as well to being affordable solutions [5]. In addition, many intelligent-hybrid approaches have been innovated to provide an efficient solution to widespread problems [7]-[9].

Advances in computer science and electronic technologies have facilitated control engineers to apply intelligent-based controllers due to [10]:

1. Design and implementation of electronic circuits with a powerful performance with information processing.

2. Significant development in simulation platforms and computer-aided software that enables control designers to build and further design various efficient conflagrations of control as well as process systems.

In 1974, Mamdani first applied fuzzy based controllers for laboratory-scale steam engines [11], a year after his work with Assilian [12] had proved the superiority of fuzzy based controllers over the fixed based controllers on DDC algorithms. Later on, the process control designers extended Mamdani's innovative work in order to design and implement various control systems to deal more efficiently with a different application of more complex realworld processes. The so-called 'Mamdani fuzzy model' was reported as intuitive with a widespread acceptance inference system. Around a decade later, Takagi and Sugeno proposed another fuzzy model that could map any smooth, nonlinear function to any prescribed precision, within any compact set. This model is presented by a set of fuzzy 'IF-THEN' rules. The rules of the so-called Takagi-Sugeno (T-S), fuzzy model characterise the local linear input-output relationships of any nonlinear system. When this proposed fuzzy model is applied, it gave a reasonable performance in a water cleaning process, as well as a converter in a steel-making process [13]. Both the Mamdani and T-S models have been successfully implemented in various applications. Comprehensive reviews have been written about Fuzzy controllers; types, effectiveness applications [5], [14]. 
Neural networks have also a remarkable approximation ability that has inspired many researchers to propose a new controller that can use the prediction performance of those networks in control configurations. One of the most interested configurations is nonlinear autoregressive moving average technique, NARMA-L2, proposed by Narendra and Mukhopadhyay [15] by introducing an efficient solution to the problems that cause slow performance of backpropagation training algorithms. The main idea of the NARMA-L2 controller is using approximate models that represent a dynamic process, by training ANN offline and then designing the NARMA-L2 controller by rearrangement the trained ANN model. The obvious advantage of the NARMA-L2 controller is that it does not require an additional submodel to be trained, as is required in other neuro-controllers, such as Model Reference Adaptive Control, MRAC, and Model Predictive Controller, MPC [16].

More recently, other new controllers have been proposed and applied in different fields. Some contributions are referred to in the following by Piltan et al. [17], who proposed and implemented a SISO fuzzy estimating sliding mode controller on a robot manipulator, as well as a PSO, used as an optimisation tool to tune and adjust the sliding function. Valikhani et al. [18] used a novel control method based on the emotional decision-making process, which occurs in mammalian brains. The so-called brain emotional learning-based intelligent controllers, BELBIC, are applied to control twice-fed induction generator wind turbine systems. Shen et al. [19] introduced a new adaptive solution to neural tracking control problems by proposing a novel neural control for a class of uncertain pure-feedback nonlinear systems.

NARMA-L2 based controllers have been recently gaining enormous interest amongst researchers in different areas. Necsulescu et al. [20] designed a MIMO NARMA-L2 controller, together with output redefinition techniques for controlling the flight of an unmanned aerial vehicle, UAV. The results showed a good and stable performance of the proposed controller. Fourati et al. [21] controlled a bioreactor with an NARMA-L2 controller and proved that the trajectory tracking performance obtained was better than with the use of the inverse neural model controller. Valluru et al. [22] implemented NARMA-L2 controller on a series of DC motors, in order to regulate speed. The performance index indicated better performance than a PID controller. Uçak et al. [23] proposed a novel NARMA-L2 controller based on online Support Vector Regression, SVR. The proposed controller was tested on a bioreactor system. Its performance compared with a PID controller. Jalil et al. [24] used an NARMA-L2 to control the vibration of a flexible beam structure, with non-collocated sensor-actuator placement.

This study proposes a new design of MIMO NARMA-L2 controller, based on FIS approximation submodels at the identification of the process to be controlled. ANFIS configuration is used into the FIS submodels and trained separately by the hybrid method (BpLSE) and Patricle Swarm Optimisation, to find the optimal parameters of the FISs. This proposed controller has been implemented, followed by testing on a binary distillation column, which exhibits nonlinear and dynamic behaviour. 


\section{Distillation Modelling}

\subsection{Process description}

Oil refineries, as well as other chemical and petrochemical plants, widely use the process of distillation. The chemical compounds in a mixture are separated into their individual component chemicals using distillation columns. These types of columns operate extensively in the petroleum, natural gas, liquid and chemical industries [25]. However, the process utilised in these columns is very energy intensive columns. The Department of Energy in the USA published a report and showing that distillation columns are the largest consumers of energy in the chemical industry. Typically, they account for $40 \%$ of the energy consumed by all petrochemical plants. Even with this high energy consumption, distillation is still widely utilised for this separation and purification method [26].

Figure 1 shows the schematic diagram of a binary distillation column. The feed mixture is separated into two products; one is a distillate or overhead, and the other is the bottom product. Heat is supplied to the column via a reboiler, in order to vaporise the liquid in the base of the column. The vapour goes up through trays inside the column to reach the top. The vapour then liquefies in the condenser. Liquid from the condenser drops into the reflux drum. Finally, the some of the distillate product is removed from this drum as a pure product. The rest of the liquid is fed back near the top of the column as reflux, while another product is produced at the bottom.

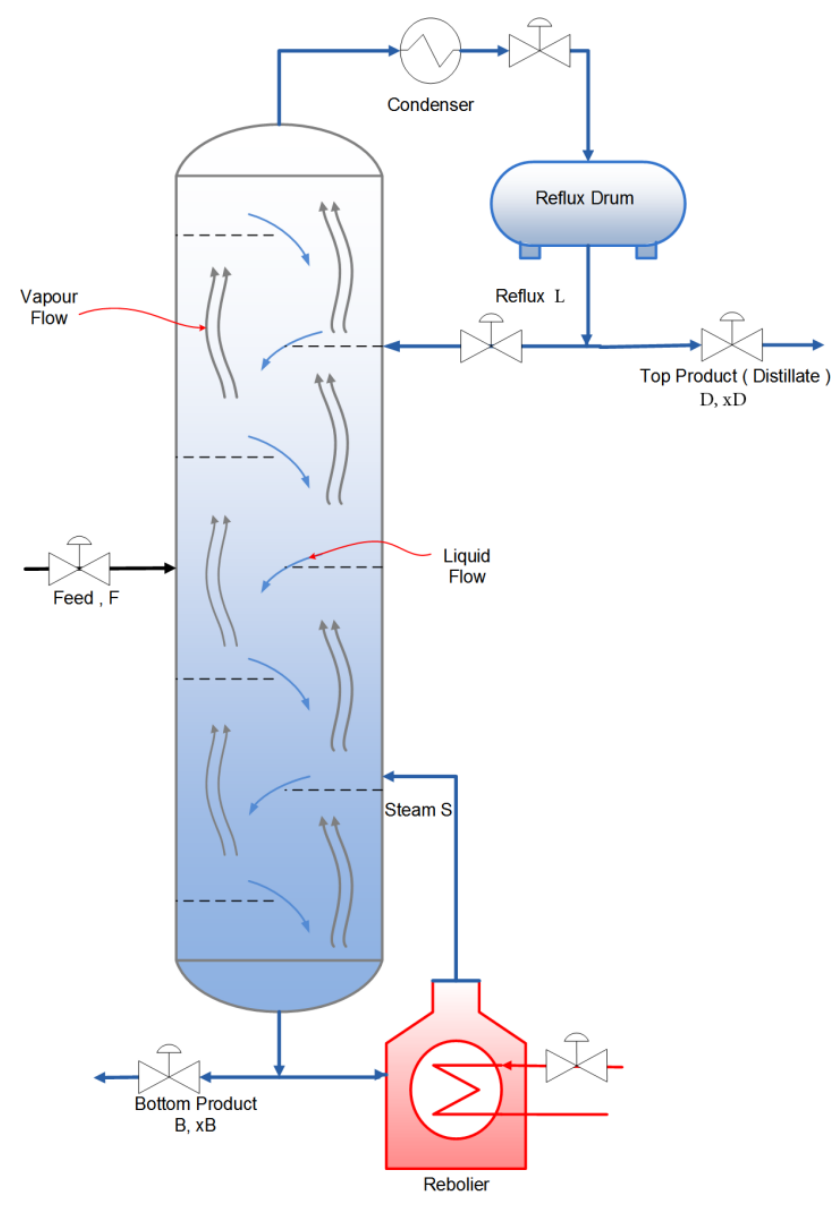




\subsection{Model representation}

The Luyben model is the basis of this binary distillation column, and modelled and simulated in this research, based on [27], [28] with the following considerations:

1) No chemical reactions occur inside the column.

2) There is constant pressure.

3) Binary mixture.

4) Constant relative volatility.

5) No vapour hold-up occurs in any stages.

6) Constant hold-up liquid at all trays.

7) Perfect mixing and equilibrium for vapour-liquid at all stages.

Hereafter, the mathematical equations of the model can be written per stage by following equations:

- On each tray (excluding reboiler, feed and condenser stages):

$$
M_{i} \frac{d x_{i}}{d t}=L_{i+1} x_{i+1}+V_{i-1} y_{i-1}-L_{i} x_{i}-V_{i} y_{i}
$$

- $\quad$ Above the feed stage $i=N_{F}+1$;

$$
M_{i} \frac{d x_{i}}{d t}=L_{i+1} x_{i+1}+V_{i-1} y_{i-1}-L_{i} x_{i}-V_{i} y_{i}+F_{v} y_{F}
$$

- Below the feed stage, $i=N_{F}$ :

$$
M_{i} \frac{d x_{i}}{d t}=L_{i+1} x_{i+1}+V_{i-1} y_{i-1}-L_{i} x_{i}-V_{i} y_{i}+F_{L} x_{F}
$$

- At the reboiler and column base, $i=1, x_{i}=\chi_{B}$ :

$$
M_{B} \frac{d x_{i}}{d t}=L_{i+1} x_{i+1}-V_{i} y_{i}+B x_{B}
$$

- $\quad$ At the condenser, $\mathrm{i}=\mathrm{N}+1, \mathrm{x}_{\mathrm{D}}=\mathrm{X}_{\mathrm{N}+1}$;

$$
M_{D} \frac{d x_{D}}{d t}=V_{i-1} y_{i-1}-L_{i} x_{D}-D x_{D}
$$

- Vapour-liquid equilibrium relationship for each tray:

$$
y_{i}=\frac{\propto x_{i}}{1+(\propto-1) x_{i}}
$$


The flow rate of constant molar flow:

Above the feed stage:

$$
L_{i}=L, \quad V_{i}=V+F_{v}
$$

At or below the feed stage:

$$
L_{i}=L+F_{L}, \quad V_{i}=V
$$

Since

$$
\begin{aligned}
& F_{l}=q_{F} \times F \\
& F_{v}=F+F_{L}
\end{aligned}
$$

The constant hold-up for both the condenser and the reboiler as:

Reboiler:

$$
\begin{aligned}
& B=L+F_{L}-V \\
& D=V+F_{V}-L
\end{aligned}
$$

The feed compositions $X_{F}$ and $y_{F}$ are found from the flash equation as:

$$
F_{z F}=F_{L} \times x_{F}-F_{V} \times y_{F}
$$

The abbreviations, operation conditions and steady states of the column are in the appendix, the schematic diagram of a theoretical stage of the column is shown in Figure 2.

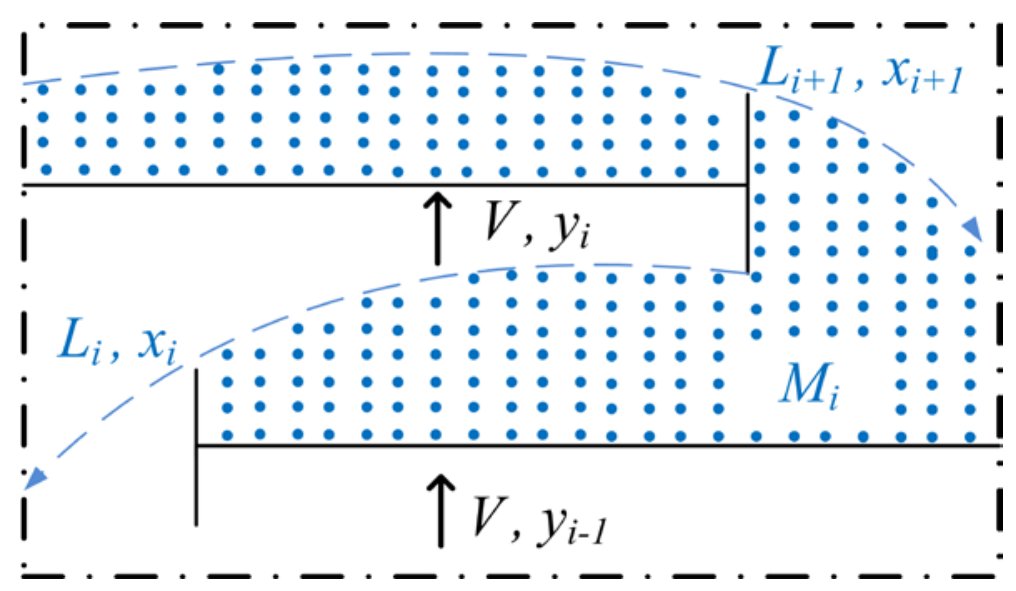

Figure 2 Schematic diagram of ith stage of a binary distillation column

\section{Process Identification}

The performance index of nonlinear process identification is measured by the accuracy of input-output mapping. This index is considered the key in the optimisation of many advanced control systems, such as neurocontrollers or fuzzy inference based controllers. 
Any process model formed by input-output pairs is typically divided into two main categories. The first one is a mathematical expression of a system model, and the other is the model identification. However, a mathematical method itself is required for simplicity and generality, in addition to the many assumptions that could be necessary [13]. Therefore, identification of a nonlinear process by mapping input-output dataset is the most favoured method for achieving high accuracy and capturing the nonlinearity of the process.

Artificial Neural Network (ANN) and the fuzzy-based method are both the most intelligent techniques employed in the process of identification, whether for modelling nonlinear systems or control configurations purposes. In this section, a theoretical introduction is presented about these approaches, which are used for the comparison purpose with the proposed controller.

\subsection{Artificial Neural Networks}

ANN is a group of nodes (neurons) that mimic the biological neural networks of the brains of animals; specifically, the neuronal-synaptic mechanisms that store, learn and retrieve information, based on only empirical data. They are basically employed in machine learning in order to identify complex functions. ANNs are considered one of the most significant subfields of artificial intelligence, showing excellent performance to learn the input-output relations of nonlinear functions (processes). Once the network has learned, by introducing enough dataset of input-output pairs, the output can be estimated faster and with better efficiency. ANN-based approaches are still being applied extensively to overcome various complications in many diverse practical applications, ranging from nonlinear system identification to adaptive control, as well as pattern recognition, image processing, medical diagnostics, process monitoring, renewable and sustainable energy and laser-based applications [29]-[31].

\subsection{Fuzzy models}

Fuzzy logic is the other major subfield of artificial intelligence, principally dealing with imprecision by emulating reasoning in the human brain, for the approximation process. Fuzzy based modelling has been explored widely in literature and applied extensively in industrial applications because its biggest advantage is that there is no need for precise quantitative analyses. It mostly depends on the IF-THEN rules, which are linguistic expressions specified by membership functions in the form: IF $\boldsymbol{X}$ THEN $\boldsymbol{Y}$, where $\boldsymbol{X}$ and $\boldsymbol{Y}$ are labels of fuzzy sets [32], whereas $\boldsymbol{Y}$ is a crisp value for the T-S models [13].

The majority of engineering problems deal with a number of input-output pairs. This is in contrast to other problems that look for the relationship between input-output in sets form. Consequently, a pre-processing, called fuzzification and defuzzification should occur before and after the fuzzy inference system, as depicted in Figure 3.

Even though there have been many successes in applications, the rule base of most of the fuzzy control systems has been static. Thus, it has to be tuned manually by an expert operator until a good performance is reached. The most challenging task facing fuzzy system designers is the computational time and effort required to develop the fuzzy parameters, including the rules and membership functions. 


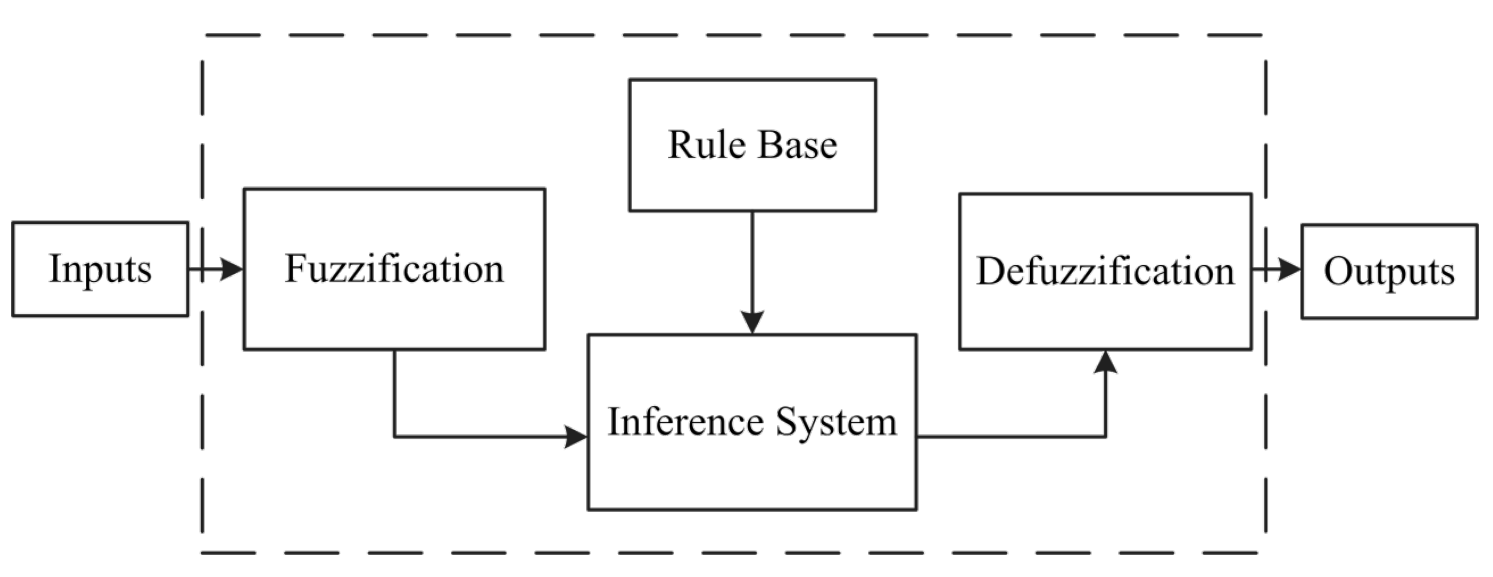

Figure 3 Basic fuzzy inference system

Therefore, many innovative solutions have been proposed to make an automatic selection of these parameters. One of the most efficient approaches is 'adaptive neuro-fuzzy' or 'adaptive network-based fuzzy inference systems' (ANFIS) [33] that uses, commonly, the hybrid backpropagation-least square error method to tune the parameters of FIS.

\subsection{Adaptive Neuro-fuzzy Inference Systems}

As mentioned in the previous section, the implementation of ANFIS introduced an automatic adjustment of the FIS parameters. There are two methods used in the ANFIS approach; backpropagation, as well as the combination of backpropagation and least square error, called a hybrid learning method.

The main motivational aims of using ANFIS are the features that present the learning capabilities of both ANN and fuzzy inference systems. The learning algorithm adjusts the membership functions of a Sugeno fuzzy model, using input-output dataset. Figure 4 shows an example of the simplified ANFIS architecture, which contains two membership functions (A, B) for both inputs ( $\left.\mathrm{x}_{1}, \mathrm{x}_{2}\right)$ and four rules as well as four membership functions of output (y).

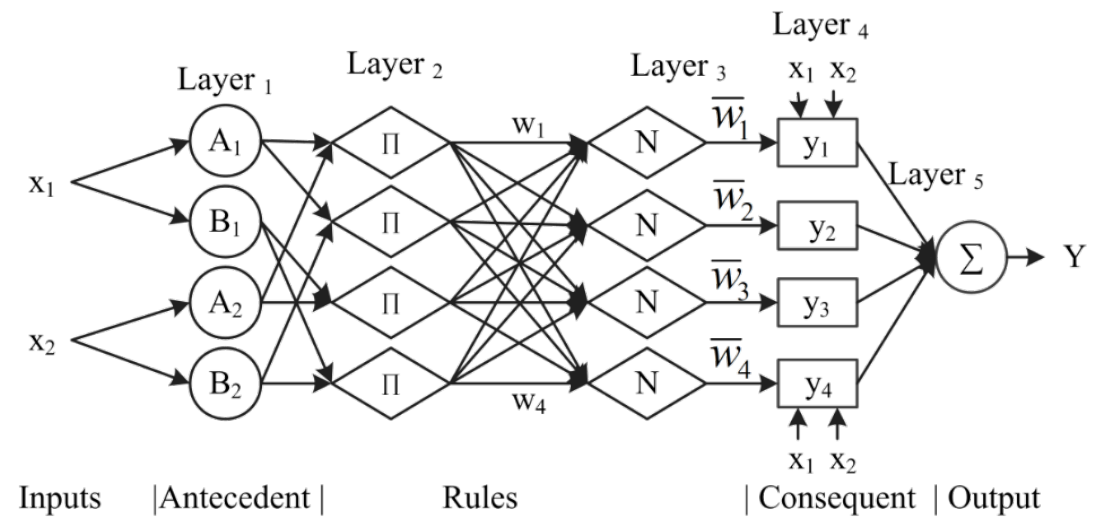

Figure 4 An ANFIS architecture of two inputs, four rules, and first order Sugeno model

According to Figure 4, $w_{1}$ to $w_{4}$, which are the weights of correctness for the rules, are calculated through T-norm. Additionally, these weights are used to compute $y_{1}, y_{2}, y_{3}$ and $y_{4}$ respectively. The final output $Y$ is expressed as: 


$$
Y=\frac{w_{1} y_{1}+w_{2} y_{2}+w_{3} y_{3}+w_{4} y_{4}}{w_{1}+w_{2}+w_{3}+w_{4}}
$$

After normalising the weights, the output is written as:

$$
Y=\bar{w}_{1} y_{1}+\bar{w}_{2} y_{2}+\bar{w}_{3} y_{3}+\bar{w}_{4} y_{4}
$$

It is reported that there is no guarantee that ANN-based learning algorithm converges and the adjusting of FIS will be successful [34]. The weaknesses relating to neural networks and fuzzy inference systems appear complementary and natural intelligence based methods could be implemented to optimise the combination to produce the best possible synergetic performance to create a hybrid intelligent system [35]. Using different intelligent-based optimisation methods for adaptation of FIS has been extensively explored [36], [37].

\subsection{Genetic Algorithm}

Genetic algorithm, GA, is a heuristic, global, optimisation likelihood search algorithm that mimics the genetic mechanisms that form the basis of the theory of Darwin's natural selection and biological evolution. Initially, this generates a random population of candidate solutions towards the optimal fitness (objective function) by performing specific techniques, such as reproduction, crossover, and mutation. The procedures are repeated until the prescribed objective function is accepted, or the pre-set number of generations is executed. GA has been extensively employed in a variety of domains with considerable efficacy in recent years, and this is primarily attributed to their almost universal relevance and promise.

\subsection{Particle Swarms Optimisation}

Kennedy and Eberhart have proposed the Particle Swarm Optimisation (PSO) in 1995 [38] and 2001 [39]. PSO algorithm is considered to be enormously successful as a swarm optimisation tool. Over the past decade, many studies have shown the advantages of the application of PSO's in a wide range of engineering problems [40].

The implementing procedures of the PSO can be detailed as follows: all particles or candidates (usually between 10 and 100) are placed at a random location and are theoretically considered to travel randomly within the search space. The direction of each particle then changes gradually to move more assuredly along the direction of its best previous position, in order to determine an even better position, according to predefined criteria or an objective function. The preliminary velocity and location of the particles are nominated randomly. The subsequent velocity can be updated by the following equation:

$$
V_{i+1}=w V_{i}+C_{1} R_{1} \times\left(P b_{i}-x_{i}\right)+C_{2} R_{2} \times\left(G b-x_{i}\right)
$$

Consequently, the position of the new particle is computed by adding the previous position to the obtained velocity, as shown in the following equation:

$$
x_{i+1}=x_{i}+V_{i+1}
$$


where $V=$ the particle's velocity, $x=$ the particle's position, $R_{1} ; R_{2}=$ independent random factors uniformly distributed from 0 to $1, C_{1} ; C_{2}=$ acceleration coefficients, $w=$ inertial weight.

Eq. 16 is used to compute the new velocity of the particle, according to its previous value and the distance of its current position from its own best position $(\mathrm{Pb})$ and the global best position (GB). Then, the particle travels to a new position in the search space, according to Eq. 17. PSO is implemented in this study to find the optimal parameters of all FIS submodels in the identification process as shown in Figure 5.

\section{NARMA model}

The Nonlinear Auto-Regressive Moving Average, NARMA, is one of the most certified representations of general discrete-time nonlinear systems. This model representation is used in the form of past, current, and the future system parameters, as shown in Eq. 18.

$$
y_{(\mathrm{k}+\mathrm{d})}=f\left[y_{(t)}, y_{(t-1)}, \ldots y_{(t-n+1)}, u_{(t)}, u_{(t-1)}, \ldots u_{(t-n+1)}\right]
$$

where $y(t), u(t)$ are the input and output of the system respectively, and $f$ [.] is a nonlinear approximation of input and output of the system.

For the identification stage, a global approximation could be employed to compute $f$ [.], such as ANN, FIS or SVM. For control purposes, using backpropagation ANN, for finding a control signal $u_{(t)}$ is noted to be quite slow because of the involving dynamic gradient methods. Therefore, an efficient method is proposed by Narendra and Mukhopadhyay by introducing approximation models to overcome computational difficulties. Two classes of NARMA model have been tested; NARMA-L1 and NARMA-L2. It was found that the second class involving two sub-approximation functions is more efficient and adequate in the identification and adaptation of control contexts [15].

$$
\begin{aligned}
\hat{y}_{(\mathrm{t}+\mathrm{d})}= & f\left[y_{(t)}, y_{(t-1)}, \ldots y_{(t-n+1)}, u_{(t-1)}, \ldots u_{(t-n+1)}\right] \\
& +g\left[y_{(t)}, y_{(t-1)}, \ldots y_{(t-n+1)}, u_{(t-1)}, \ldots u_{(t-n+1)}\right] \times u_{(t)}
\end{aligned}
$$

The two sub-functions, $f$ and $g$, are used in the identification phase as well as to compute the control signal as follows:

$$
u_{(t)}=\frac{y_{(\mathrm{k}+\mathrm{d})}-f\left[y_{(t)}, y_{(t-1)}, \ldots y_{(t-n+1)}, u_{(t)}, u_{(t-1)}, \ldots u_{(t-n+1)}\right]}{g\left[y_{(t)}, y_{(t-1)}, \ldots y_{(t-n+1)}, u_{(t)}, u_{(t-1)}, \ldots u_{(t-n+1)}\right]}
$$

Eq. 19 and 20 represent single-input-single-output SISO systems. As reported in the literature, using ANN-based estimation methods have a noticed disadvantage; they are expected to get trapped in local minima. Therefore, the models approximated through ANN will be accepted only locally; this is the primary motivation behind choosing an alternative approach to approximate NARMA submodels. The key advantage of ANFIS compared to backpropagation-based identification approaches is that reasoning and learning in the uncertain 
and vague situations are guaranteed; henceforth the proposed model prevalent in operation ranges is attained precisely.

Thus, our proposed control system design, using FIS as approximation functions. So, we can rewrite the equation 19 as:

$\left[\begin{array}{l}y_{1(t+2)} \\ y_{2(t+2)}\end{array}\right]=\left[\begin{array}{l}\operatorname{FIS}_{1}\left(y_{1(t-1)}, y_{1(t)}, y_{1(t+1)}, y_{2(t-1)}, y_{2(t)}, y_{2(t+1)}, u_{1(t-1)}, u_{1(t)}, u_{1(t+1)}, u_{2(t-1)}, u_{2(t)}, u_{2(t+1)}\right) \\ \operatorname{FIS}_{4}\left(y_{1(t-1)}, y_{1(t)}, y_{1(t+1)}, y_{2(t-1)}, y_{2(t)}, y_{2(t+1)}, u_{1(t-1)}, u_{1(t)}, u_{1(t+1)}, u_{2(t-1)}, u_{2(t)}, u_{2(t+1)}\right)\end{array}\right]$
$+\left[\begin{array}{l}F I S_{2}\left(y_{1(t-1)}, y_{1(t)}, y_{1(t+1)}, y_{2(t-1)}, y_{2(t)}, y_{2(t+1)}, u_{1(t-1)}, u_{1(t)}, u_{1(t+1)}, u_{2(t-1)}, u_{2(t)}, u_{2(t+1)}\right) \\ \operatorname{FIS}_{5}\left(y_{1(t-1)}, y_{1(t)}, y_{1(t+1)}, y_{2(t-1)}, y_{2(t)}, y_{2(t+1)}, u_{1(t-1)}, u_{1(t)}, u_{1(t+1)}, u_{2(t-1)}, u_{2(t)}, u_{2(t+1)}\right)\end{array}\right] \times u_{1(t+2)}$
$+\left[\begin{array}{l}\text { FIS }_{3}\left(y_{1(t-1)}, y_{1(t)}, y_{1(t+1)}, y_{2(t-1)}, y_{2(t)}, y_{2(t+1)}, u_{1(t-1)}, u_{1(t)}, u_{1(t+1)}, u_{2(t-1)}, u_{2(t)}, u_{2(t+1)}\right) \\ \text { FIS }_{6}\left(y_{1(t-1)}, y_{1(t)}, y_{1(t+1)}, y_{2(t-1)}, y_{2(t)}, y_{2(t+1)}, u_{1(t-1)}, u_{1(t)}, u_{1(t+1)}, u_{2(t-1)}, u_{2(t)}, u_{2(t+1)}\right)\end{array}\right] \times u_{2(t+2)}$

where the subscripts 1 and 2 of $u$ and $y$ represent the first and second input or output respectively, and $t$ is a time step. The schematic diagram of the time series identification process of MIMO systems is depicted in Figure 5. FIS1 and FIS4 represent the relationship between the

\subsection{Identification results}

Obtain an NARMA model of the system to be controlled is the first step, in order to design the proposed controller later. This is done by mapping the input-output pairs. The dataset was collected by simulating the binary distillation column by introducing 40 distributed random values, each lasting 50 sampling time for $(\mathrm{L})$ and $(\mathrm{V})$ to collect product compositions, $\mathrm{xD}, \mathrm{xB}$ of different situations. The distillate composition was approximately between 0.95 and 1 (mole fraction), while the bottom composition was around 0.005 to 0.12 (mole fraction). A total of 2000 datasets were collected for the identification stage.

Figure 6 shows the block diagram of the modelled binary distillation column which is simulated using MATLAB ${ }^{\circledR}$ and Simulink ${ }^{\circledR}$; the model was built via representing the mathematical equations mentioned earlier in section 2.2. Simulink ${ }^{\circledR}$ is considered the very powerful graphical design tool to model, analyse and visualise of a system response which is easily manipulated to facilitate the control experiments. In order to collect sufficient information for creating a good model that represents the system behaviour, the inputs are selected as PRBS (Pseudo-Random Binary Sequence) within the operating range. The collected dataset has been presented in Figure 7 showing the inputs and outputs of the simulated column.

The obtained dataset has been divided into 70\% and 30\% for training, and testing subsets respectively. For ANFIS structure, the hybrid method (Bp-LSE) has been applied to find the optimal parameters of FISs of the proposed model and then PSO is employed to see if we can get better (optimised) FISs parameters. 


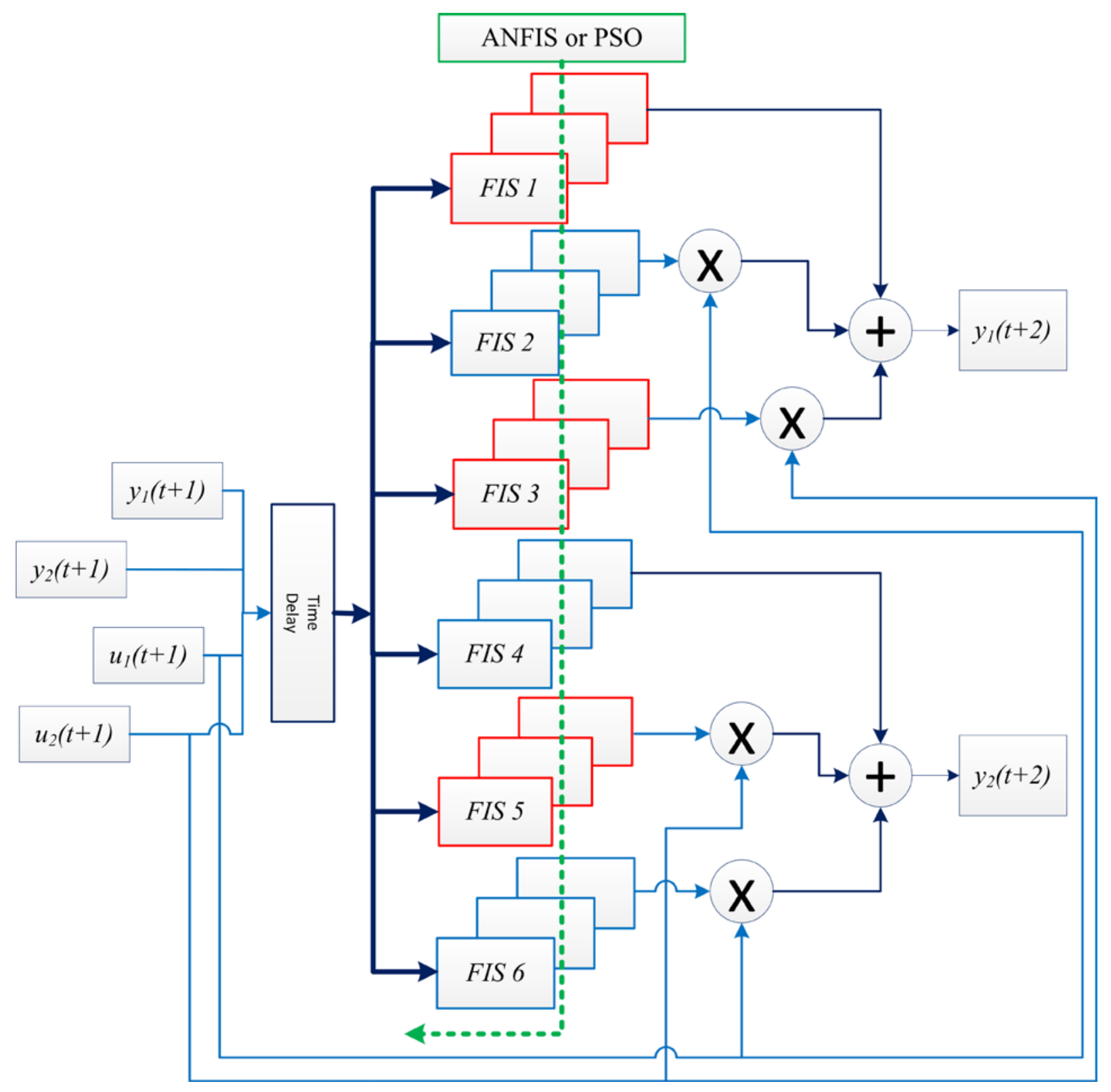

Figure 5 Schematic diagram of the proposed MIMO NARMA-L2 model

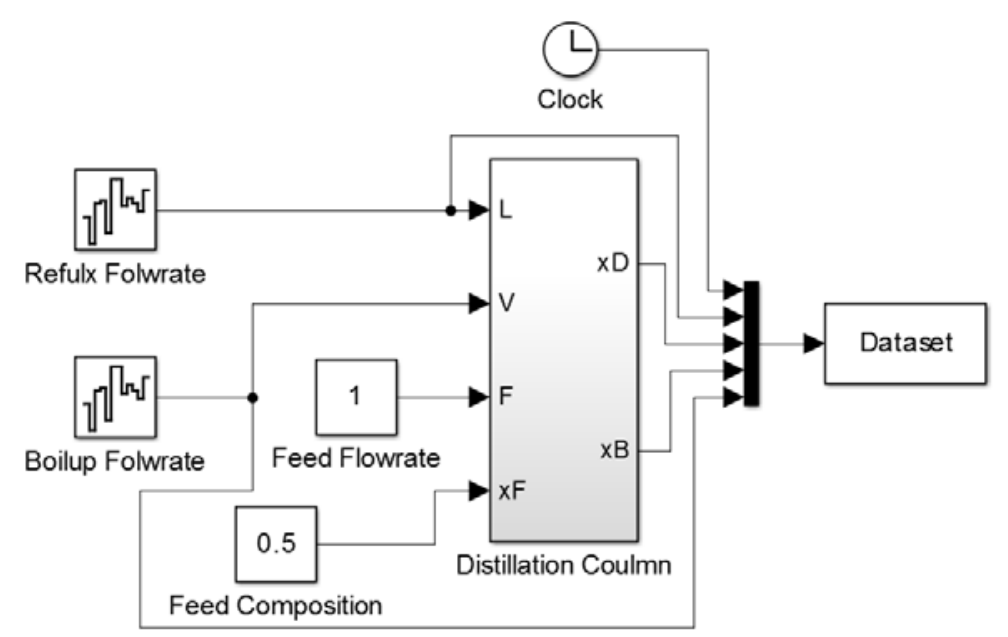

Figure 6 Figure 6 Simulation model of a binary distillation column 

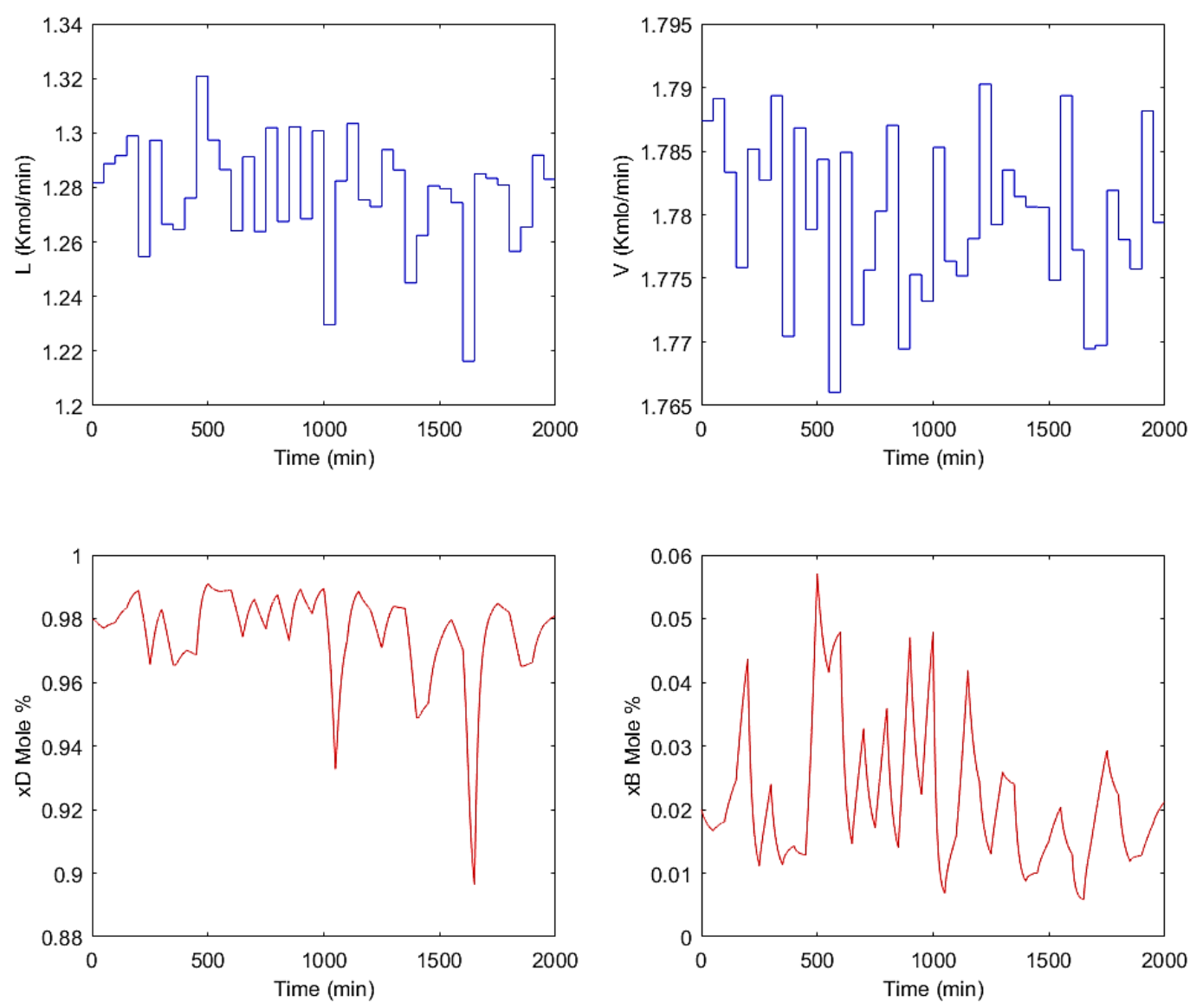

Figure 7 Inputs/outputs of the simulation of the distillation

The identification performance index is measured using commonly used statistical criteria regarding the Root Mean Squared Errors (RMSE) between the predicted and actual output(s) as expressed in equation (22):

$$
R M S E=\sqrt{\frac{1}{n} \sum_{i=1}^{n}\left(\widehat{Y}_{l}-Y_{i}\right)^{2}}
$$

where $\widehat{Y}$ is a vector of $n$ predictions, $Y$ is representing the vector of actual values corresponding to the inputs to the model which produced the approximation. This prediction performance index is also used as an objective function to compute the convergence of PSO. Due to the random initialization of the location of the particle, PSO was run 20 times to explore a broad coverage of the search space. 


$$
\left[\begin{array}{l}
Y_{r_{1}} \\
Y_{r_{2}}
\end{array}\right]=\left[\begin{array}{l}
F I S_{1} \\
F I S_{4}
\end{array}\right]+\left[\begin{array}{l}
F I S_{2} \\
F I S_{5}
\end{array}\right] \times U_{1(t+2)}+\left[\begin{array}{l}
F I S_{3} \\
F I S_{6}
\end{array}\right] \times U_{2(t+2)}
$$

\begin{tabular}{|c|c|c|c|c|}
\hline \multirow{2}{*}{ Learning method } & \multicolumn{2}{|c|}{ RMSE (xD) } & \multicolumn{2}{c|}{ RMSE (xB) } \\
\cline { 2 - 5 } & Training & Testing & Training & Testing \\
\hline Hybrid (BP and LSE) & 0.0044312 & 0.01072 & 0.014566 & 0.01217 \\
\hline PSO & $7.6 \times 10^{-6}$ & $4.873 \times 10^{-5}$ & $1.3216 \times 10^{-5}$ & $9.4261 \times 10^{-6}$ \\
\hline
\end{tabular}

It is clear that, as shown in Table 1, the FISs submodels are better optimised using PSO compared to the hybrid method with less RMSE. The visual comparison between the actual and predicted outputs for the both methods is shown in Figure 8 for training and testing cases. ANFIS tuned by PSO model shows adequately accurate predictions for distillate and bottoms compositions, while the ANFIS trained by the hybrid method lack of adequate prediction especially for the second output in both training and testing subsets.
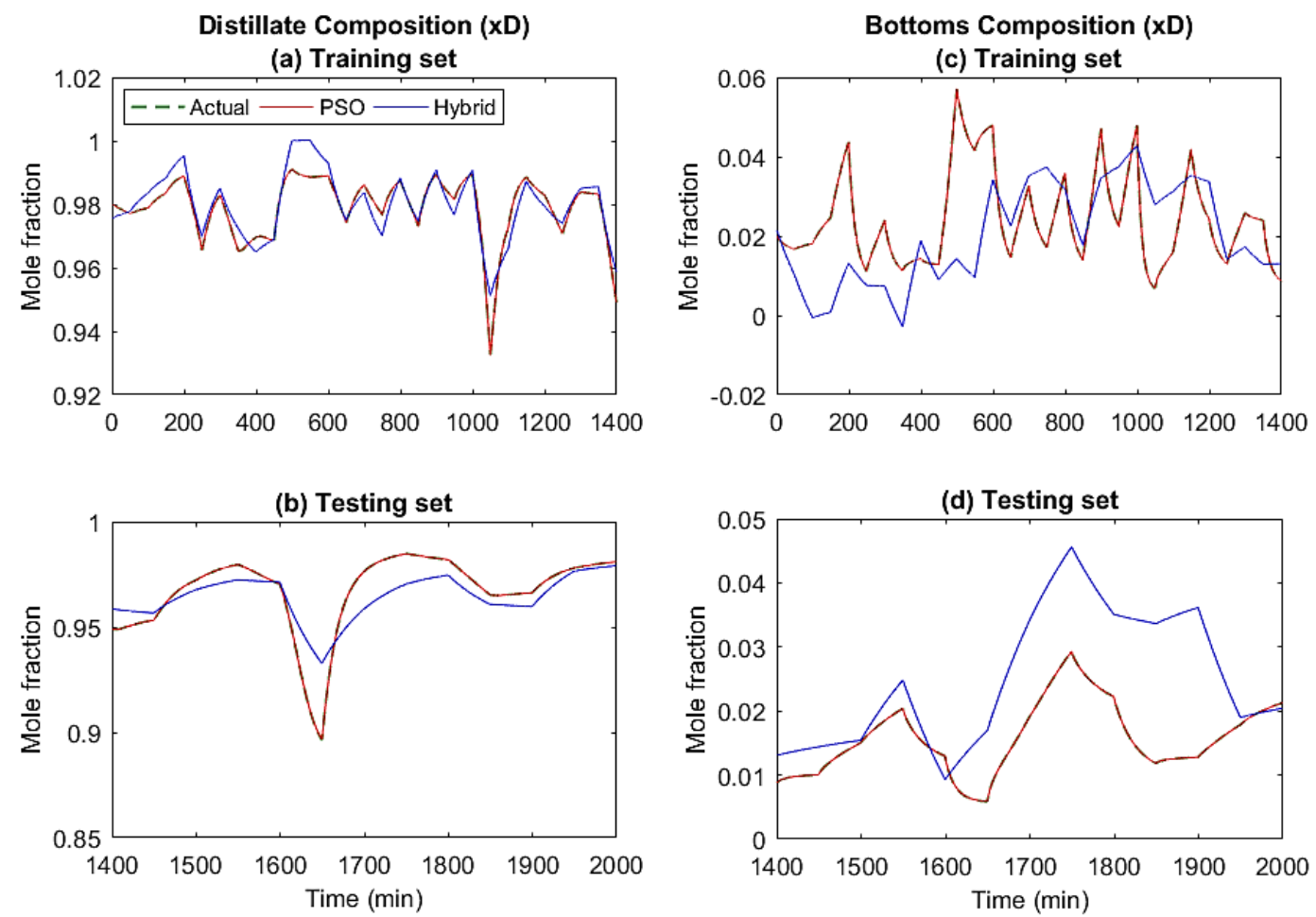

Figure 8 Prediction model behaviour vs. actual outputs (distillate and bottoms compositions), training and testing subsets,

\section{NARMA-L2 controller}

After accepting the generalisation of the modelling of the proposed configuration, testing of the model can be done with the unseen data (test set). The trained FISs can be rearranged to form the MIMO NARMA-L2 controller. The control signal calculated as: 


$$
\begin{gathered}
\left(\left[\begin{array}{l}
Y_{r_{1}} \\
Y_{r_{2}}
\end{array}\right]-\left[\begin{array}{l}
F I S_{1} \\
F I S_{4}
\end{array}\right]\right)=\left[\begin{array}{l}
F I S_{2} \\
F I S_{5}
\end{array}\right] \times U_{1(t+2)}+\left[\begin{array}{l}
F I S_{3} \\
F I S_{6}
\end{array}\right] \times U_{2(t+2)} \\
\left(\left[\begin{array}{l}
Y_{r_{1}} \\
Y_{r_{2}}
\end{array}\right]-\left[\begin{array}{l}
F I S_{1} \\
F I S_{4}
\end{array}\right]\right)=\left[\begin{array}{ll}
F I S_{2} & F I S_{3} \\
F I S_{5} & F I S_{6}
\end{array}\right]\left[\begin{array}{l}
U_{1(t+2)} \\
U_{2(t+2)}
\end{array}\right] \\
{\left[\begin{array}{l}
U_{1(t+2)} \\
U_{2(t+2)}
\end{array}\right]=\left[\begin{array}{ll}
F I S_{2} & F I S_{3} \\
F I S_{5} & F I S_{6}
\end{array}\right]^{-1} \times\left(\left[\begin{array}{l}
Y_{r_{1}} \\
Y_{r_{2}}
\end{array}\right]-\left[\begin{array}{l}
F I S_{1} \\
F I S_{4}
\end{array}\right]\right)}
\end{gathered}
$$

where $Y_{r 1}$ and $Y_{r 2}$ are the set points or desired compositions of the column products. Figure 9 is a schematic diagram of the proposed controller.

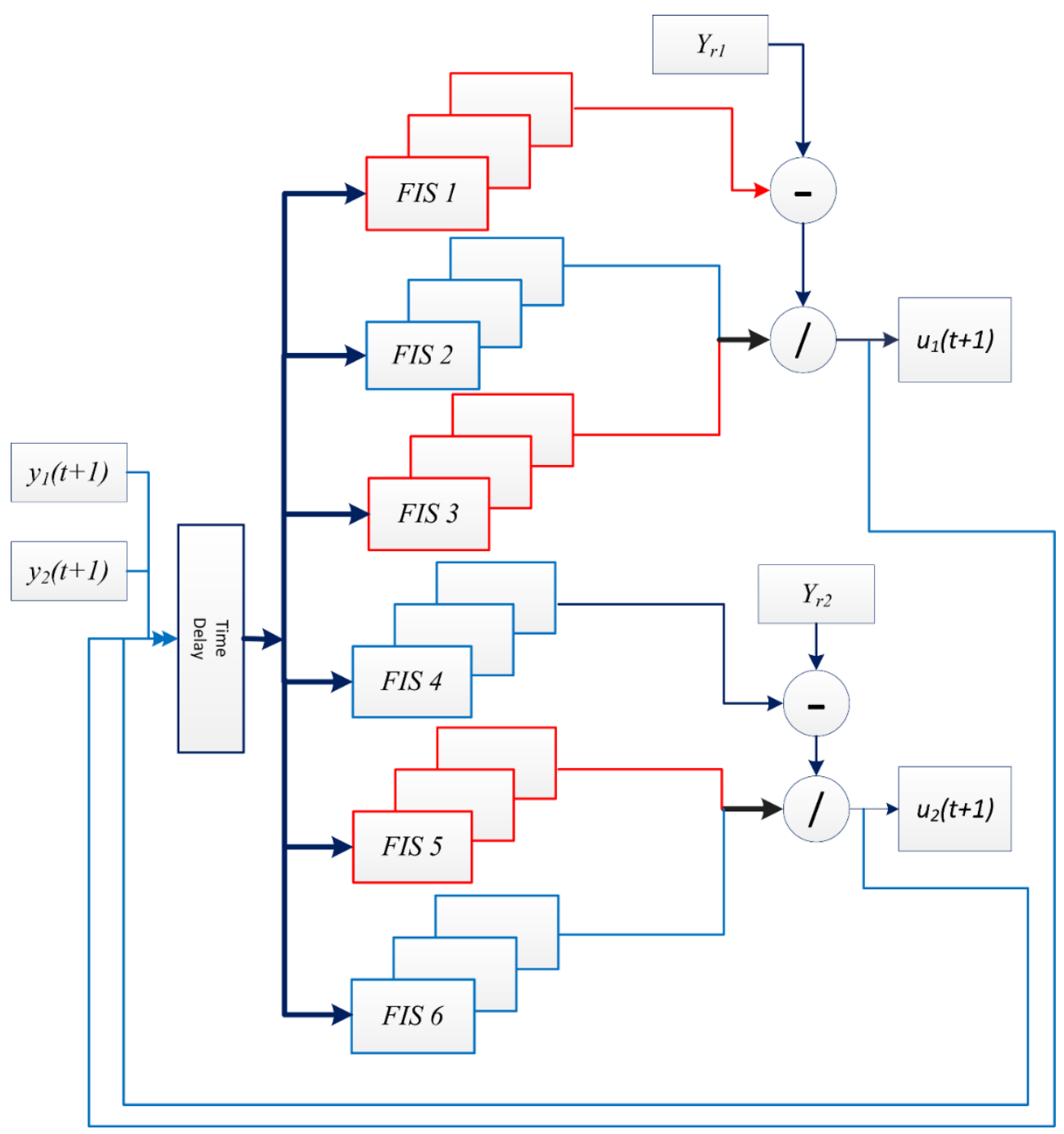

Figure 9 ANFIS based MIMO NARMA-L2 control configuration

For comparison purposes, various control configurations have been applied to control the column. These control configurations are:

1. Traditional PID 
2. PD, PI and PID-like Fuzzy logic controller tuned by PSO and GA separately

3. NARMA-L2 based on ANN

ANN-based NARMA-L2 applied here, which is available in Simulink ${ }^{\circledR}$ library in MATLAB $^{\circledR}$ platform from The MathWorks, Inc., uses Multi-Layer Perceptron (MLP), with a symmetric sigmoid transfer function in the hidden layers, and linear transfer as activation function in the output layer. All ANN default parameters have been employed in this context.

The performance index of the different controllers with various tuning methods is given in Table 2. The time response is shown in Figure 10; it is noticeable that all of the designed controllers use different configurations passed the transient response requirements. Nevertheless, the performance of the MIMO ANFIS-based NARMA-L2 indicated better attainment regarding the performance index, as well as better transit responses in addition to eliminating the loop interactions.

Table 2 Comparison of performance index of the proposed controller vs. other controllers

\begin{tabular}{|c|c|c|c|c|}
\hline \multirow{2}{*}{ Controller } & \multicolumn{2}{|c|}{ Tuning / Configuration } & \multicolumn{2}{|c|}{ Performance Index } \\
\cline { 3 - 5 } & \multicolumn{2}{|c|}{ PSO } & 0.0015 & 18.5396 \\
\hline \multirow{2}{*}{ PID } & \multirow{2}{*}{ PSO } & PD like & 0.0018 & 26.6249 \\
\cline { 3 - 5 } & & PI like & 0.0047 & 37.2752 \\
\cline { 3 - 5 } & \multirow{2}{*}{ FLC } & PID like & 0.0011 & 10.0091 \\
\cline { 3 - 5 } & \multirow{2}{*}{ GA } & PD like & 0.0092 & 57.1706 \\
\cline { 3 - 5 } & \multirow{2}{*}{ PI like } & 0.0042 & 33.2897 \\
\cline { 3 - 5 } & & PID like & 0.0014 & 11.4034 \\
\hline NARMA-L2 & \multirow{2}{*}{ ANN } & 0.0006 & 4.0751 \\
\hline NARMA-L2 & \multirow{2}{*}{ PSO_ANFIS } & $\mathbf{0 . 0 0 0 2 1}$ & 3.289 \\
\hline
\end{tabular}

To check the robustness and stability of the proposed controller, the desired compositions of the column are set to change asynchronously, to examine the process behaviour regarding inputs variance played as disturbances that should be rejected and should handle it well. The desired compositions of distillate and bottoms produced are changed every 100 min for up to a thousand minutes. It can be observed that the control actions of the controller can be adapted to eliminate the effects of the input variance, where the convergence of the desired responses can be achieved after the adaptation of the control output, as shown in Figure 10. 


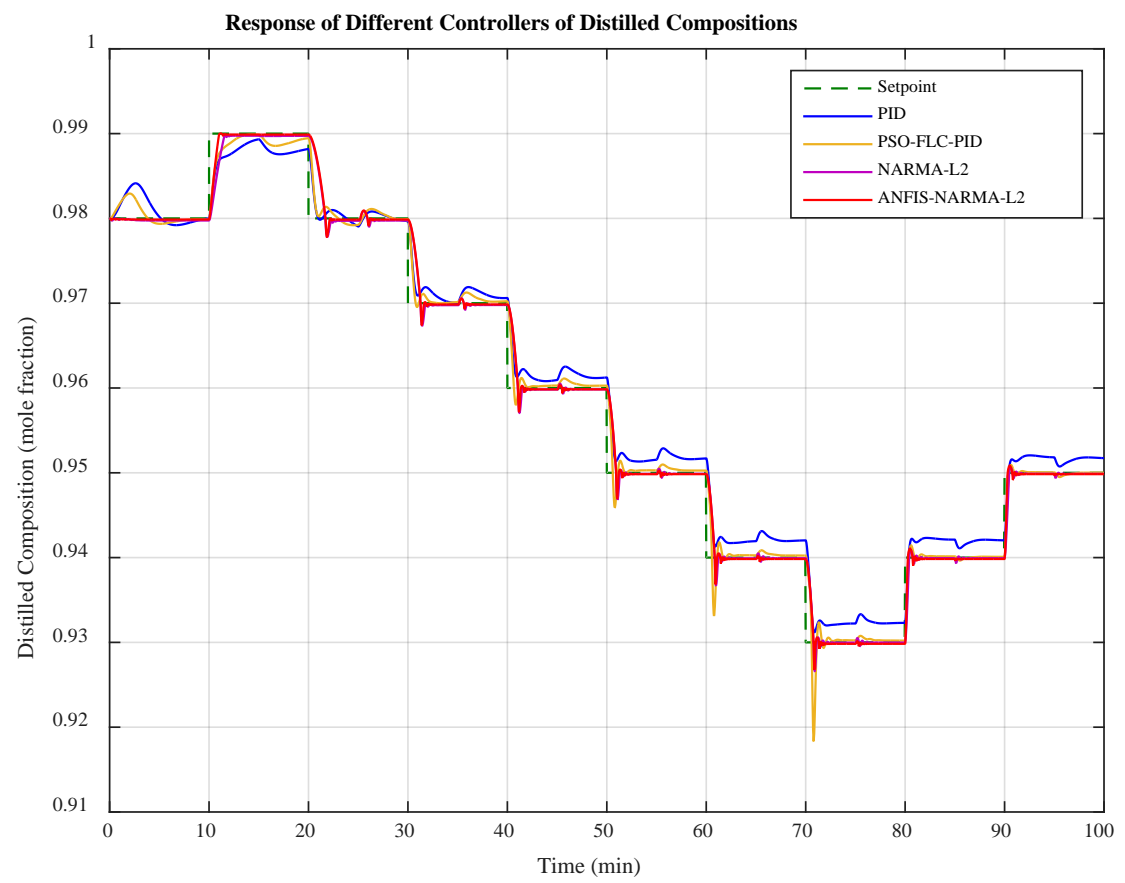

(a)

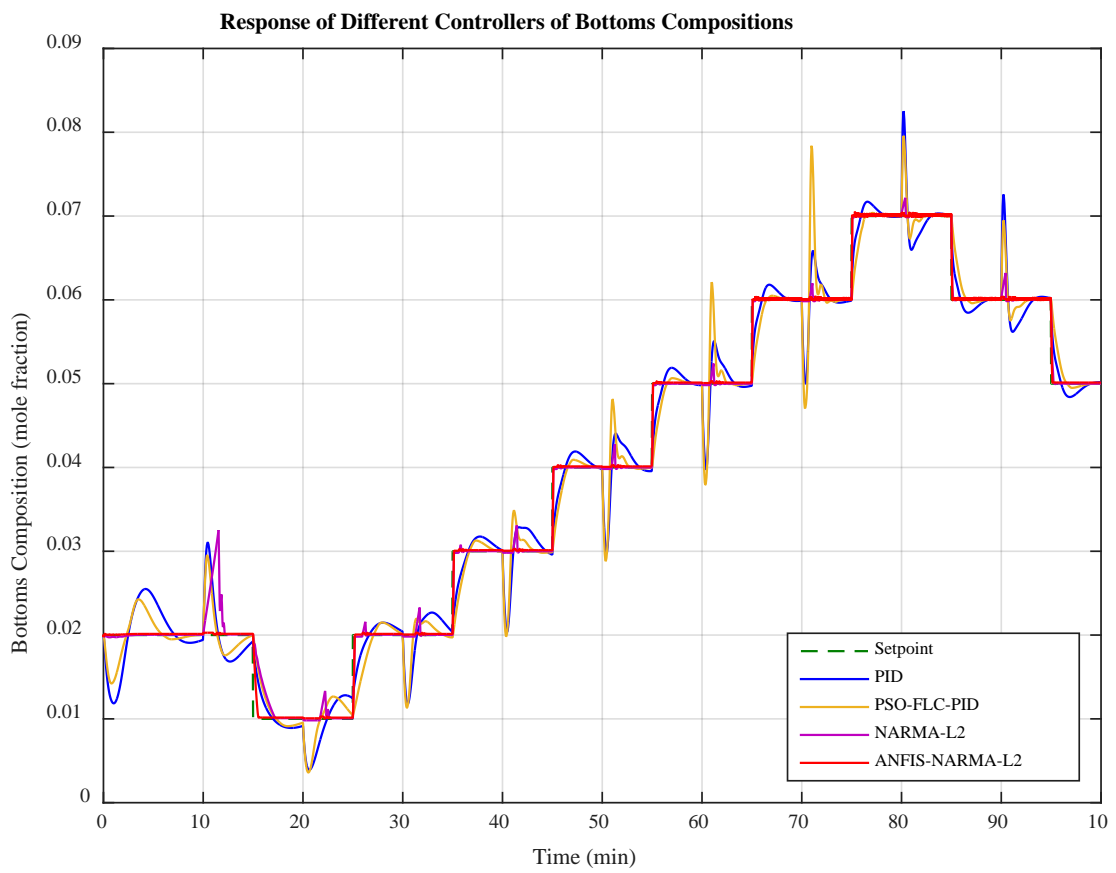

(b)

Figure 10 Time response of the various controllers, (a) distillate composition, (b) bottoms composition 


\section{Conclusions}

This study focussed on a proposal for a new MIMO ANFIS-based NARMA-L2 controller, in which ANFIS is employed to construct the configuration of FIS submodels of NARMA-L2. Initially, an ANFIS-based NARMA-L2 model is erected to estimate the system outputs using the hybrid learning method (backpropagation and least square error). In addition, the scholastic search method, particle swarm optimisation, implemented to find the optimum inference system parameters of the submodels. The generality of the obtained model is then tested using unseen data to ensure the model is sufficiently unbiased. The proposed controller is designed by rearranging the obtained submodels to compute the required control signal to achieve the desired performance.

A binary distillation column is used as a benchmark in order to evaluate the proposed modelling and control system. The manipulated variables are the boilup and reflux flow rates of the column used to control the product compositions. The accomplishment of the controller is compared with different control configurations include conventional PID, PD, PI and PIDlike Fuzzy logic controller tuned by PSO and GA separately, as well as NARMA-L2 based on ANN. The proposed controller outperformed all these configurations, in terms of performance index (ISE and ITAE), overshoot and steady state error.

The stability and robustness of the controller against input variance have also been examined, by selecting asynchronous setpoints. The simulation results showed that the proposed controller is powerful in achieving low tracking error, time response behaviour, as well as the elimination of input interactions.

\section{Acknowledgment}

The corresponding author is grateful to the Iraqi Ministry of Higher Education and Scientific Research for supporting the current research.

\section{References}

[1] B. a. Ogunnaike, "Problems and challenges of industrial process control: a commercialpolymerization reactor case study,” Proc. IEEE Symp. Comput. Control Syst. Des., pp. 27-36, 1994.

[2] T. Samad and A. Annaswamy, "the Impact of Control Technology," IEEE Control Syst. Soc., vol. 1, p. 246, 2011.

[3] M. Mahfouf, S. Kandiah, and D. Linkens, "Fuzzy model-based predictive control using an arxstructure with feedforward,” Fuzzy Sets Syst., vol. 125, no. 1, pp. 39-59, 2002.

[4] A. Gani, A. Siddiqa, S. Shamshirband, and F. Hanum, “A survey on indexing techniques for big data: taxonomy and performance evaluation,” Knowl. Inf. Syst., vol. 46, no. 2, pp. 241-284, Feb. 2016.

[5] R.-E. Precup and H. Hellendoorn, “A survey on industrial applications of fuzzy control,” Comput. Ind., vol. 62, no. 3, pp. 213-226, Apr. 2011.

[6] M. Chandrasekaran, M. Muralidhar, C. M. Krishna, and U. S. Dixit, “Application of soft computing techniques in machining performance prediction and optimization: a 
literature review,” Int. J. Adv. Manuf. Technol., vol. 46, no. 5-8, pp. 445-464, Jan. 2010.

[7] S. Shamshirband, N. B. Anuar, M. L. M. Kiah, V. A. Rohani, D. Petković, S. Misra, and A. N. Khan, "Co-FAIS: Cooperative fuzzy artificial immune system for detecting intrusion in wireless sensor networks,” J. Netw. Comput. Appl., vol. 42, pp. 102-117, Jun. 2014.

[8] C. Caraveo, F. Valdez, and O. Castillo, "Optimization of fuzzy controller design using a new bee colony algorithm with fuzzy dynamic parameter adaptation,” Appl. Soft Comput., vol. 43, no. C, pp. 131-142, Jun. 2016.

[9] L. Amador-Angulo, O. Mendoza, J. Castro, A. Rodríguez-Díaz, P. Melin, and O. Castillo, "Fuzzy Sets in Dynamic Adaptation of Parameters of a Bee Colony Optimization for Controlling the Trajectory of an Autonomous Mobile Robot," Sensors, vol. 16, no. 9, p. 1458, Sep. 2016.

[10] T. Samad and G. Balas, Software-Enabled Control: Information Technology for Dynamical Systems. John Wiley \& Sons, 2003.

[11] E. H. Mamdani, “Application of fuzzy algorithms for control of simple dynamic plant,” Proc. Inst. Electr. Eng., vol. 121, no. 12, p. 1585, 1974.

[12] E. H. Mamdani and S. Assilian, "An experiment in linguistic synthesis with a fuzzy logic controller,” Int. J. Man. Mach. Stud., vol. 7, no. 1, pp. 1-13, Jan. 1975.

[13] T. Takagi and M. Sugeno, "Fuzzy identification of systems and its applications to modeling and control,” Syst. Man Cybern. IEEE Trans., vol. SMC-15, no. 1, pp. 116132, 1985.

[14] K. Tai, A.-R. El-Sayed, M. Biglarbegian, C. Gonzalez, O. Castillo, and S. Mahmud, "Review of Recent Type-2 Fuzzy Controller Applications," Algorithms, vol. 9, no. 2, p. 39, Jun. 2016.

[15] K. Narendra and S. Mukhopadhyay, "Adaptive control using neural networks and approximate models,” Neural Networks, IEEE ..., 1997.

[16] O. De Jesus, A. Pukrittayakamee, and M. T. Hagan, "A comparison of neural network control algorithms," in IJCNN'01. International Joint Conference on Neural Networks. Proceedings (Cat. No.01CH37222), 2001, vol. 1, pp. 521-526.

[17] F. Piltan, A. Jalali, and N. Sulaiman, "Novel artificial control of nonlinear uncertain system: design a novel modified PSO SISO Lyapunov based fuzzy sliding mode algorithm,”... J. Robot. ..., 2011.

[18] M. Valikhani and C. Sourkounis, "A novel intelligent controller for DFIG-based wind turbine system,” in 2014 IEEE International Energy Conference (ENERGYCON), 2014, pp. 44-50.

[19] Q. Shen, P. Shi, T. Zhang, and C.-C. Lim, "Novel neural control for a class of uncertain pure-feedback systems.," IEEE Trans. neural networks Learn. Syst., vol. 25, no. 4, pp. 718-27, Apr. 2014.

[20] D. Necsulescu, Y.-W. Jiang, and B. Kim, "Neural network based feedback linearization control of an unmanned aerial vehicle," Int. J. Autom. Comput., vol. 4, no. 1, pp. 71-79, Jan. 2007.

[21] F. Fourati, S. Baklouti, and H. Moalla, "NARMA-L2 neural control of a bioreactor," in 2015 4th International Conference on Systems and Control (ICSC), 2015, pp. 504-509.

[22] S. K. Valluru, M. Singh, and N. Kumar, "Implementation of NARMA-L2 Neuro 
controller for speed regulation of series connected DC motor," in 2012 IEEE 5th India International Conference on Power Electronics (IICPE), 2012, pp. 1-7.

[23] K. Uçak and G. Öke Günel, “A Novel Adaptive NARMA-L2 Controller Based on Online Support Vector Regression for Nonlinear Systems,” Neural Process. Lett., Feb. 2016.

[24] N. A. Jalil and I. Z. M. Darus, "NARMA-L2 Vibration Controller for Flexible Structure with Non-collocated Sensor-Actuator," in 2013 Fifth International Conference on Computational Intelligence, Modelling and Simulation, 2013, pp. 17-22.

[25] C. L. Smith, Distillation Control: An Engineering Perspective. Wiley, 2012.

[26] W. L. Luyben, "Design and control of dual condensers in distillation columns," Chem. Eng. Process. Process Intensif., vol. 74, pp. 106-114, 2013.

[27] W. L. Luyben, Process Modeling, Simulation and Control for Chemical Engineers, 2nd ed. McGraw-Hill Book Company, 1999.

[28] W. Luyben, Distillation design and control using Aspen simulation. John Wiley \& Sons, Inc., 2013.

[29] F. Amato, A. López, E. M. Peña-Méndez, P. Vaňhara, A. Hampl, and J. Havel, "Artificial neural networks in medical diagnosis," J. Appl. Biomed., vol. 11, pp. 47-58, 2013.

[30] S. Mondal, A. Bandyopadhyay, and P. K. Pal, “Application of artificial neural network for the prediction of laser cladding process characteristics at Taguchi-based optimized condition,” Int. J. Adv. Manuf. Technol., pp. 2151-2158, 2013.

[31] M. Maarouf, A. Sosa, B. Galván, D. Greiner, G. Winter, M. Mendez, and R. Aguasca, "The Role of Artificial Neural Networks in Evolutionary Optimisation: A Review," in Advances in Evolutionary and Deterministic Methods forDesign, Optimization and Control in Engineering and Sciences, ComputationalMethods in Applied Sciences 36, vol. 36, Springer International Publishing Switzerland 2015, 2015, pp. 209-223.

[32] J.-S. R. Jang, “ANFIS: adaptive-network-based fuzzy inference system,” IEEE Trans. Syst. Man. Cybern., vol. 23, no. 3, pp. 665-685, 1993.

[33] J. Jang, "Fuzzy Modeling Using Generalized Neural Networks and Kalman Filter Algorithm," in Proceedings of the 9th National Conference on Artificial Intelligence, 1991, pp. 762-767.

[34] a Abraham, “Adaptation of fuzzy inference system using neural learning,” Fuzzy Syst. Eng., vol. 83, pp. 53-83, 2005.

[35] a Abraham, "Hybrid intelligent systems: evolving intelligence in hierarchical layers," Do Smart Adapt. Syst. Exist., vol. 179, pp. 159-179, 2005.

[36] Y. Al-Dunainawi and M. F. Abbod, "Evolutionary Based Optimisation of Multivariable Fuzzy Control System of a Binary Distillation Column," in UKSim-AMSS 18th International Conference on Computer Modelling and Simulation, 2016, pp. 127-132.

[37] O. Cordón, F. Herrera, F. Hoffmann, and L. Magdalena, Genetic Fuzzy Systems: Evolutionary Tuning and Learning of Fuzzy Knowledge Bases, vol. 19. WORLD SCIENTIFIC, 2001.

[38] R. Eberhart and J. Kennedy, “A new optimizer using particle swarm theory,” MHS'95. Proc. Sixth Int. Symp. Micro Mach. Hum. Sci., pp. 39-43, 1995.

[39] R. C. Eberhart, Y. Shi, and J. Kennedy, Swarm intelligence. 2001. 
[40] Y. Zhang, S. Wang, and G. Ji, “A comprehensive survey on particle swarm optimization algorithm and its applications,” Math. Probl. Eng., vol. 2015, pp. 1-38, 2015.

\section{Appendix}

Abbreviations, the operating conditions and technical aspects of the distillation column are detailed in the following table.

\begin{tabular}{|c|c|c|c|}
\hline Symbol & Description & Value & Unit \\
\hline $\mathrm{N}$ & Number of trays & 20 & - \\
\hline NF & Feed stage location & 11 & - \\
\hline $\mathrm{F}$ & Typical inlet flow rate to the column & 1 & $\mathrm{kmol} / \mathrm{min}$ \\
\hline $\mathrm{D}$ & Typical distillate flow rate & 0.5 & $\mathrm{kmol} / \mathrm{min}$ \\
\hline B & Typical bottoms flow rate & 0.5 & $\mathrm{kmol} / \mathrm{min}$ \\
\hline $\mathrm{zF}$ & Light component in the feed (mole fraction) & 0.5 & - \\
\hline $\mathrm{qF}$ & Mole fraction of the liquid in the feed & 0.5 & - \\
\hline $\mathrm{L}$ & Typical reflux flow rate & 1.28 & $\mathrm{kmol} / \mathrm{min}$ \\
\hline V & Typical boil-up flow rate & 1.78 & $\mathrm{kmol} / \mathrm{min}$ \\
\hline$\alpha$ & Relative volatility & 2 & - \\
\hline $\mathrm{xD}$ & Distillate composition (mole fraction) & 0.98 & - \\
\hline $\mathrm{xB}$ & Bottoms composition (mole fraction) & 0.02 & - \\
\hline $\mathrm{i}$ & Stage number during distillation & - & - \\
\hline $\mathrm{x}$ & Mole fraction of light component in liquid & - & - \\
\hline $\mathrm{y}$ & Mole fraction of light component in vapour & - & - \\
\hline M & Tray hold-up liquid & 0.5 & kmol \\
\hline MD & Condenser hold-up liquid & 0.5 & kmol \\
\hline MB & Reboiler hold-up liquid & 0.5 & kmol \\
\hline
\end{tabular}

